\title{
CRITICAL REVIEW OF MAIN COSMOGONIC THEORIES
}

\author{
Dan Brasoveanu, Ph. D. \\ Systems Engineering Group, 9861 Broken Land Pkwy, suite 350C, \\ Columbia MD 21046, USA \\ Dan.brasoveanu@segmail.com
}

\begin{abstract}
Big Bang theory postulates the red shift of light coming from distant galaxies is caused by the Doppler Effect. Like Plato's astronomy, Big Bang theory has to be continuously propped up by countless patches in order to deal with new observations. Plato's astronomy required countless epicycles within epicycles. Big Bang requires patches such as dark energy, dark matter, cosmic inflation, accelerating expansion of the Universe and countless revisions of Hubble's constant. In addition, this theory and the fundamental law of mass and energy conservation are mutually exclusive; according to Big Bank all energy and mass appeared suddenly out of nothing. There are of course Big Bang versions that try not to contradict the conservation law. Such theories postulate a multi-verse populated by an infinite number of Universes or an infinite number of Universe instances. In other words, either the most basic law of physics or Occam's razor must be rejected in the most outrageous manner possible because otherwise the Doppler Effect cannot be used to explain the cosmic red shift.

For these reasons, some astronomers promoted the stationary Universe theory and assumed that photons lose a significant amount of energy while traversing intergalactic distances and therefore suffer a red shift. This assumption is the basis of all Tired Light theories. According to Tired Light, photon energy loss is caused by collisions with other objects such as gas molecules or dust. As a result, this theory was quickly dismissed, because this loss mechanism cannot explain the broadening of supernova spectra, the variable rate of photon frequency decay and the fact that the image of distant objects is not blurred. Subsequently other versions of Tired Light theories were ignored because supposedly any other loss mechanism is not compatible with GTR field equations. According to these equations, when the photon structure is ignored, the energy momentum-tensor of a photon moving freely through space is constant. Experiments with laser beams show this conclusion is not exactly true. Therefore even free photons actually lose some minute amount of energy. What physical mechanism may explain this loss? A simple experiment with a common object will immediately reveal this mechanism. Internal interactions are the main mechanism responsible for the gradual dampening of spring oscillations and loss of energy, not collisions with other objects. When damping of photon oscillations caused by internal friction is considered, a new version of Tired Light can be formulated; a version that is validated by all astronomic observations without recourse to any patches and without rejecting the law of energy and mass conservation or Occam's razor.
\end{abstract}

\section{Indexing terms/Keywords}

Big Bang, Tired Light, Cosmogony, Age of Universe, Quantum Mechanics, Dampened Oscillations

\section{Academic Discipline And Sub-Disciplines}

Physics, Astronomy, Quantmum Mechanics

\section{SUBJECT CLASSIFICATION}

Library of Congress Classification: Subclass QB and QC

\section{TYPE (METHOD/APPROACH)}

Analysis

\section{INTRODUCTION}

\section{STATIC INFINITE UNIVERSE}

According to the static universe model, which is also known as a "stationary" or "infinite" or "static infinite" model, the universe is both spatially infinite and temporally infinite, and space is neither expanding nor contracting. Such a universe has no spatial curvature; i.e., it is 'flat' or Euclidean. This model was first proposed by Thomas Digges [1] and was endorsed by Newton. The static infinite model is supported by large scale structures of the Universe such as "Superclusters", "Great Walls" and "Great Attractors". Given these features, the Universe has to be at least at least 80 billion to 250 billion old. For example "Coma", the largest supercluster extends up to 100 Mega parsecs ! Nevertheless the static infinite model has been abandoned, because at first glance such a model seems incompatible with the cosmic red shift discovered by Hubble and the existence of light elements. Infinite age means all matter (if any) that was not captured by black holes should consist only of iron and heavier elements because stars had plenty of time to consume lighter elements. 


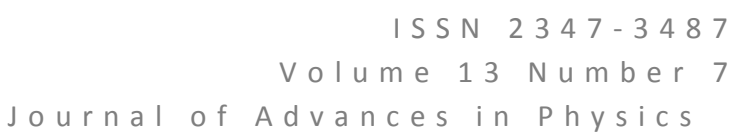

\section{HUBBLE'S LAW AND TRANSIENT UNIVERSE MODELS}

In 1929, Hubble discovered a correlation between the red shift of light coming from extra-galactic nebulae and distance to these objects [2]. Hubble assumed this nebulae move away from Earth and therefore the red shift is caused by the Doppler Effect. This assumption forms the foundation of modern transient Universe models.

The Doppler explanation of red shift leads to the conclusion that the Universe is transient and at some time in the past, all matter was concentrated into a singularity, which exploded. This explosion is called the Big Bang. How was the singularity formed? According to the standard Big Bang theory, the singularity came out of ... nowhere [Ref 3], i.e., the law of mass and energy conservation is also transient. Furthermore, as will be shown later, Big Bang requires the rejection or amendment of yet other laws of physics.

Discarding the law of mass end energy conservation was seen as a problem by some experts, which therefore proposed other versions of the Big Bang theory. According to one such version, which is inspired by ancient Indian myths, the Universe is engaged in an endless cycle of deaths by Big Crunches and re-births by Big Bangs [3]. According to another, our Universe was formed as a quantum bubble, one of countless others encompassed in a multiverse [4].

Big Bang versions proliferated due to new astronomical observations that invalidated previous versions. Astronomical observations indicate the rate of red shift decreases with distance. According to the Big Bang paradigm this proves the Universe expansion is accelerating. To explain this acceleration, never versions of Big Bang assume dark matter and dark energy [3] although dark matter and energy have not been observed and most likely are unobservable due to properties attributed to these entities. Dark matter and dark energy were also invoked to explain the anisotropy of cosmic background radiation [3]. To explain the large scale structures of the Universe, the fact that the Universe has the same temperature in all directions although there was insufficient time to exchange information between distant cosmic regions, the lack of high energy relic particles that an arbitrary hot and dense Universe would produce and the zero spatial curvature, an inflationary period of evolution was assumed and in order to explain inflation, a hypothetical inflationary field [5] was concocted. In addition, the speed of light in vacuum was supposed to be greater than $299,792 \mathrm{~km} / \mathrm{s}$ during this period. To summarize, Big Bang versions are either incompatible with known physics, with Occam's razor [6] or with both.

\section{TIRED LIGHT}

In 1929, Zwicky proposed a different red shift mechanism, which is compatible with an infinite and eternal Universe [7]: photons traveling vast distances lose energy by collision with interstellar gas, dust or other photons, or through some other mechanism. Because an energy loss leads to increased wavelength, the loss causes a red shift. If the loss is caused by collisions with interstellar matter the red shift should be roughly proportional to the distance traveled.

The Tired Light name was coined by Tolman [8]. Tired Light was abandoned by the late 1990s for allegedly failing a series of astronomical tests:

1) According to previous papers [9, 10 and 11] comparing surface brightness of galaxies with red shift, in a simple (static and Euclidian) universe, light received from an object drops proportional to the square of object distance and the apparent area of the object also drops proportional to the square of the distance, so the surface brightness (light received per surface area) would be constant, regardless of distance. Astronomical observations show brightness decreases with distance

2) Due to interactions with matter causing the red shift, photon paths would be perturbed (allegedly, no other phenomena could explain the energy loss). As a result, distant objects would appear fuzzy. Astronomical observations do not reveal such fuzziness.

3) Tired Light cannot explain the broadening of light curves of distant supernovas.

4) An infinite old Universe would contain no light elements. Due to fusion reactions, only iron and heavier elements should be left, that is if black holes would not have captured all matter. In reality light elements abound, especially hydrogen.

5) Tired light does not explain why the rate of red shift increases with distance. Note: According to Big Bang this is caused by the acceleration of Universe expansion.

6) The spectrum of cosmic background radiation matches well the spectrum of a blackbody with a temperature of approx. 3K. The spectrum of a star deviates significantly from that of a black body. The combined spectrum of several stars is also significantly different.

\section{REVISED TIRED LIGHT}

Assuming loss of photon energy proportional to distance, Tired Light is falsified by test no. 1, 2 and 5 . For these reasons, Zwicky also proposed a non-linear law of energy loss [7]:

$$
E(x)=E_{0} \exp \left(-\frac{x}{R_{0}}\right) \text { (eq. 1) }
$$

where $E(x)$ is the photon energy at distance $x$ from source, $E_{0}$ is the initial photon energy, and $R_{0}$ is a constant called resistance of space. 
The value of $R_{0}$ can be determined as follows:

1) From the Doppler effect equation

$\frac{f}{f_{0}}=\frac{c}{c+v}=\frac{1}{1+z}$ (eq. 2)

where $v$ is the source velocity, $c$ is the speed of light in vacuum, $f$ is the red shifted frequency, $f 0$ is the initial frequency and $z \equiv v / c$ is the red shift.

2) From eq. 1 and 2:

$\frac{E}{E_{0}}=\frac{f}{f_{0}}=\frac{1}{1+z}=\exp \left(-\frac{x}{R_{0}}\right)$ (eq. 3)

3) According to recent measurements [12], Hubble's constant, $H=71.9 \mathrm{Km} / \mathrm{s} / \mathrm{MPA}$, where MPA (mega parsec) $=3.0857 \mathrm{E} 19 \mathrm{~km}$. This means,

$$
H=\frac{71.9}{3.0857 \mathrm{E} 19}=2.3301 \mathrm{E}-18 \mathrm{~s}^{-1} \text {. }
$$

4) Therefore

$$
\frac{1}{1+71.9 / e E 5}=\exp \left(\frac{-1 M P A}{R_{0}}\right)(\text { eq. } 4)
$$

5) From eq. $4, \mathrm{R}_{0}=1.2877 \mathrm{E} 23 \mathrm{~km}$.

Therefore, eq. 1 becomes

$$
E(x)=E_{0} \exp \left(-\frac{x}{1.2877 E 23}\right)(\text { eq. 5) }
$$

Because $c z \approx H x$, eq. 5 can be also written as

$$
E(x)=E_{0} \exp \left(-\frac{c z / H}{1.2877 E 23}\right)(\text { eq. } 6)
$$

From eq. 6:

$E(x)=E_{0} \exp \left(-\frac{3 e 5 z}{1.2877 E 23 \cdot 2.3301 E-18}\right)=E_{0} \exp (-0.9998 z)$ (eq. 6)

Photon energy at distance $\mathrm{x}$ can also be expressed as a function of time:

$$
E(x)=E_{0} \exp \left(-\frac{x}{1.2877 E 23}\right)=E_{0} \exp \left(-\frac{c t}{1.2877 E 23}\right)=E_{0} \exp \left(-2.3297 \cdot 10^{-18} \cdot t\right)(\text { eq. } 7)
$$

\subsection{Revised Tired Light and the First Astronomical Test}

A more careful analysis shows that Tired Light is not falsified by the first Astronomical tests. If the energy of each photon is red shifted according to eq. 6 , the energy of light received scaled by surface area, i.e., the brightness is also reduced and the brightness reduction factor $>\frac{1}{\exp (-0.9998 z)}=\exp (0.9998 \mathrm{z})$ because brightness loss is caused not just by a reduction of energy of photons but also by absorption and deflection of photons as a result of interactions with interstellar matter. Assuming the loss caused by these interactions is equal to $2 z$, the overall reduction factor is:

$$
\text { reduction factor }=\exp (0.9998 z)+2 z \text {. (eq. 8) }
$$

The values of this factor calculated for $z$ between 0.05 and 0.9 are listed in Table 1 . According to astronomical observations the reduction factor is approximately equal to $(1+z)^{n}$ and the exponent $n$ has values between 2.2 and 3.7 [9]. The wide range of exponent values is due to the fact that several galaxy clusters were observed in two different bands. The term $2 z$ is an average value. Each extra galactic object is characterized by an individual term, which depends on galaxy direction. In conclusion, Table1 shows Tired Lights predictions match brightness observations. Lubin and Sandage [Ref 9] did not disprove Tired Light, merely proved an incomplete understanding of intergalactic phenomena. 


\subsection{Revised Tired Light and the Second Astronomical Test}

If the interaction of photons with interstellar matter is the main cause of red shift, images of distant galaxies should be fuzzy. Astronomical observations show images of distant galaxies are clear. The second astronomical test falsifies Tired Light versions that attribute red shift to interactions of photons with other particles.

\subsection{Revised Tired Light and the Third Astronomical Test}

The absolute magnitude of stars decreases with distance as shown by eq. 8 . The light curve of supernova Type la SN 2001el [13] and the light curve of an identical twin located further away are compared in Fig. 1. If the distance to the more distant star reduces the absolute magnitude by $25 \%$ compared to supernova la 2001, the light curve of the former is visibly flattened, i.e., seems broader. To show this broadening more clearly, Fig. 2, compares the light curve of the more distant star with the light curve la SN 2001 offset by 4.8 magnitudes. As shown in these figures, the broadening of light curves of distant supernova is a simple side effect of red shift. The third astronomical test does not falsify any version of Tired Light.

Table 1. Predicted and observed brightness reduction factors

\begin{tabular}{|l|l|l|l|l|l|l|}
\hline$z$ & 0.05 & 0.1 & 0.3 & 0.5 & 0.7 & 0.9 \\
\hline $\exp (0.9998 z)+2 z$ & 1.15 & 1.31 & 1.95 & 2.65 & 3.41 & 4.26 \\
\hline$(1+z)^{2.2}$ & 1.11 & 1.23 & 1.78 & 2.44 & 3.21 & 4.10 \\
\hline$(1+z)^{3.0}$ & 1.16 & 1.33 & 2.20 & 3.38 & 4.91 & 6.86 \\
\hline$(1+z)^{3.7}$ & 1.20 & 1.42 & 2.64 & 4.48 & 7.12 & 10.75 \\
\hline
\end{tabular}

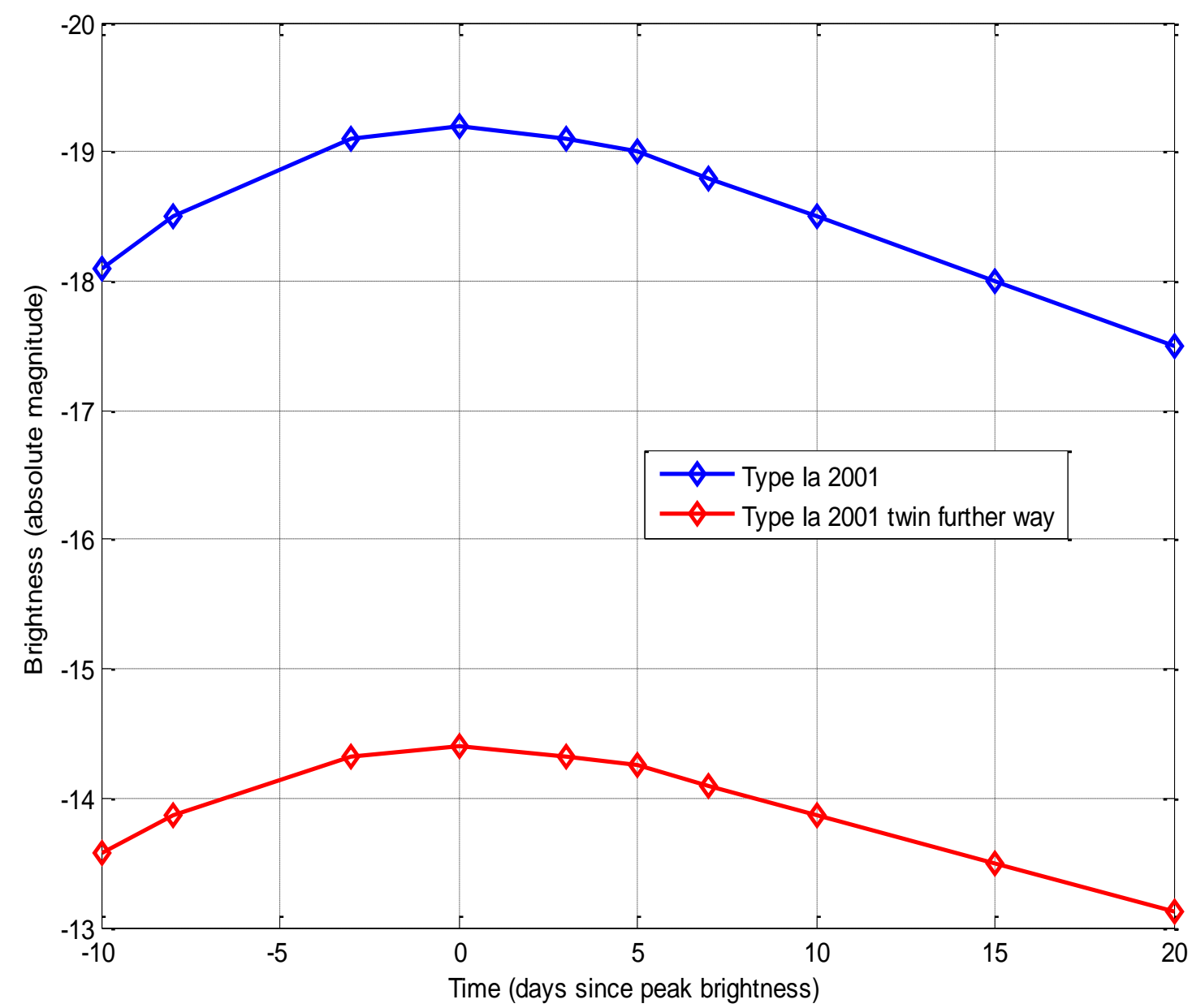

Figure 1: Light curves of supernova la 2001 and of an identical twin located further away 


\subsection{Revised Tired Light and the Fourth Astronomical Test}

Matter falling into a black hole is not trapped for ever. Black holes lose mass through Hawking radiation and eventually evaporate completely. Black holes emit neutrinos, photons, muons, gravitons and other building blocks of matter [14]. These particles then combine forming light elements; therefore matter is recycled in the Universe. Hawking's work shows the preponderance of light elements and an infinity old Universe are not mutually exclusive. Any Tired Light version satisfies the fourth astronomical test.

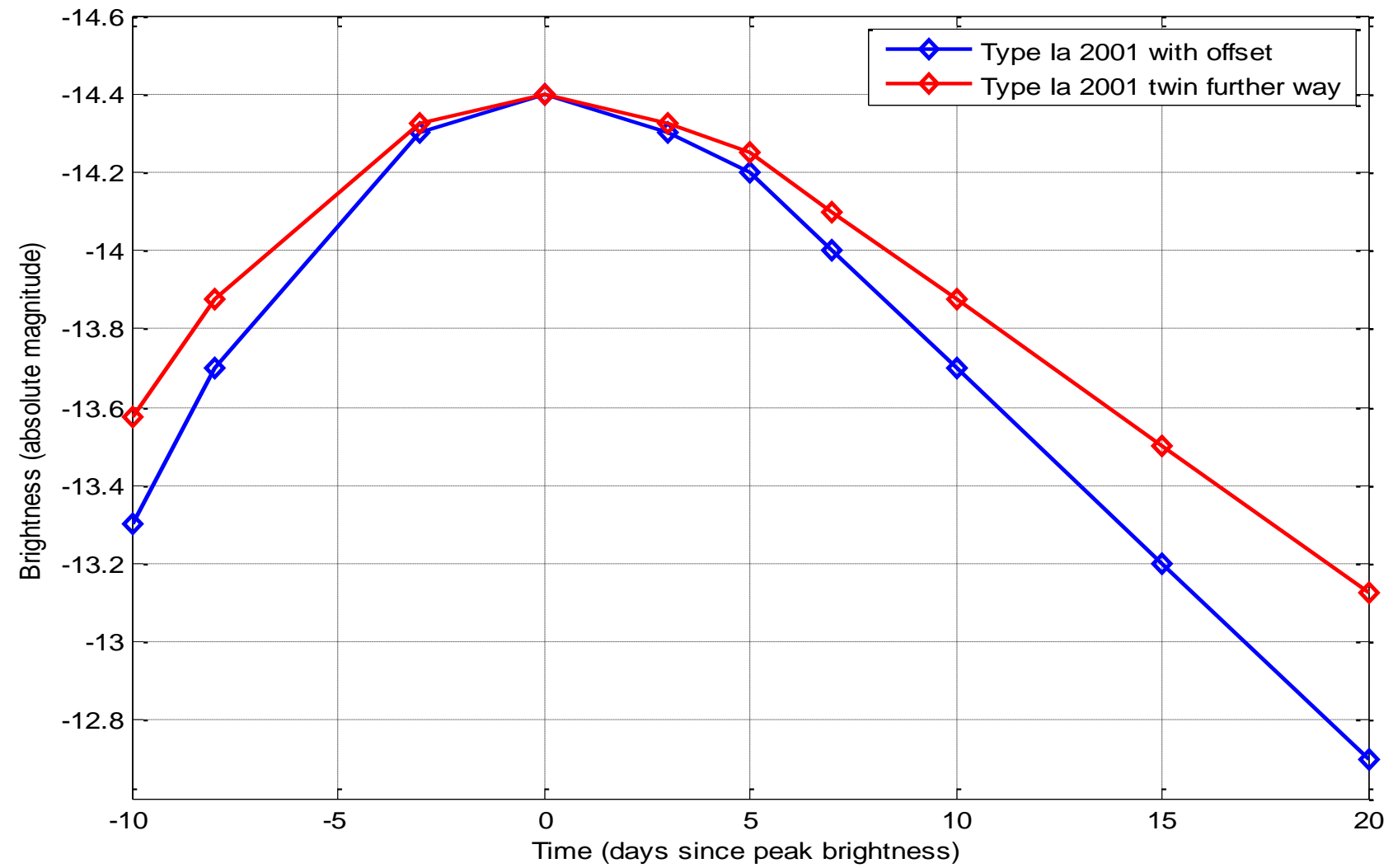

Figure 2: Light curves of supernova la 2001 offset by 4.8 magnitudes and of an identical twin located further away

\subsection{Revised Tired Light and the Fifth Astronomical Test}

When red shift is interpreted as a Doppler effect, eq. 2 and 7 yield:

$$
\frac{1}{1+z}=\exp \left(-2.3297 \cdot 10^{-18} \cdot t\right)(\text { eq. } 9)
$$

By definition

$$
z \equiv \frac{v}{C}(\text { eq. 10) }
$$

Assuming velocity is proportional to distance:

$$
v \equiv H \cdot X(\text { eq. 11) }
$$

From eq. 9, 10 and 11 :

$$
x=1.2875 \cdot 10^{23} \exp \left(2.3297 \cdot 10^{-18} t\right)(\text { eq. } 12)
$$

Eq. 12 shows the derivative of distance with respect to time is positive. As a result the Universe appears to be expanding. The second derivative, $d^{2} x / d t^{2}$ is also positive, therefore the expansion appears to be accelerating. The fifth astronomical test does not falsify the Tired Light model based on eq. 5 .

\subsection{Revised Tired Light and the Sixth Astronomical Test}

The cosmic background radiation is formed from the light of countless stars not just a few. This combination matches well the spectrum of a black body. Therefore the sixth astronomical test falsifies neither version of Tired Light. 


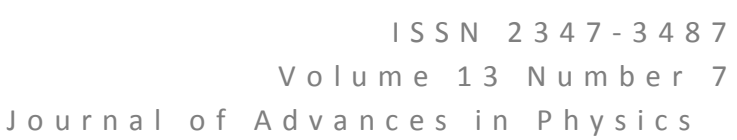

\section{PREMATURE REJECTION OF TIRED LIGHT}

Zwicky and all other supporters of Tired Light assumed that red shift is caused by interactions of photons with other particles, i.e., by external forces. This assumption is falsified by the second astronomical test. As a result, Tired Light was abandoned despite agreement with al but one astronomical test.

This rejection is premature. Tired Light should not be discarded before investigating if processes occurring inside photons may cause the red shift. The sum of internal forces in a system is null. Therefore, such forces do not change the path of a moving system. Images of distant galaxies are expected to remain crystal clear if red shift is caused by internal processes, i.e., if the photon loss of energy occurs without the involvement of any external forces.

\section{GRAVITATIONAL EFFECTS OF LIGHT}

If photons are treated as "elementary particles", then according to the General Theory of Relativity (GTR) [15], photons propagating freely do not generate gravitational fields because the invariant of the energy-momentum tensor of the electromagnetic field is a null scalar [16]. Yet massive cosmic objects bend the path of light. According to Newton's law of Universal attraction:

$\mathrm{F}=\mathrm{G} \frac{\mathrm{m}_{1} \mathrm{~m}_{2}}{\mathrm{r}^{2}}$ (eq. 13)

where, $F$ is the force of attraction between masses, $m_{1}$ and $m_{2}, G$ is the gravitational constant $\left(6.674 \times 10-11 \mathrm{~N} \cdot(\mathrm{m} / \mathrm{kg})^{2}\right)$ and $r$ is the distance between objects.

If the mass of photons would be zero then the force of attraction exerted on photons would be zero and cosmic objects regardless of mass would not bend the path of light rays. If photons have mass, then according to eq. 13, photons create gravitational fields. Is there a contradiction between GTR and Newton's law, or the invariant of the energy-momentum tensor of the electromagnetic field is not a null scalar after all? The answer to these questions is provided by a series of papers on laser beams. According to both theoretical and experimental studies $[17,18,19]$ free traveling photons emit gravitational fields. The physical mechanism responsible for these emissions can be understood considering the structure of electromagnetic radiation. Electromagnetic waves are synchronized oscillations of electric and magnetic fields. The planes of oscillations are perpendicular, therefore photons are quadrupole oscillators and quadrupole oscillators generate gravitational fields [20]. This is a result of GTR and has been validated experimentally by astronomical observations of binary stars. The motion of binary stars rotating around the common center of mass is a quadrupole oscillation and therefore generates gravitational waves. The system formed by binary stars losses energy due to these gravitational emissions until the two stars merge, a fact confirmed by Abbott's astronomical observations [21]. Therefore according to both GTR and experimental data, gravitational emissions causes a loss of energy - as should be expected based on the law of energy conservation. As demonstrated above, free traveling photons emit gravitational waves due to strictly internal processes and as a result lose energy without interaction with any other objects. Because the mechanism of gravitational emission involves only internal interactions and because in a system the sum of internal forces is zero, the path of photons is not changed by energy loss. To summarize, gravitational emissions are the cause of photon red shift and when this fact is taken into account a new version of Tired Light emerges. This new version satisfies all astronomical tests, even those supposedly falsifying Tired Light, is validated by all recent studies of gravitational effects of light, agrees completely with Occam's razor and explains cosmic features such as "Superclusters", "Great Walls", "Great Attractors" and lack of space curvature, in a simple, straightforward manner: the Universe is infinite and eternal. This new version is compatible with all established laws of physics, without recourse to ad-hoc patches such as dark matter, dark energy and inflation fields.

\section{THE LAWS OF DAMPENED OSCILLATIONS}

A review of theory of dampened oscillations is required in order to understand why results based on eq. 5 and $7 \mathrm{match}$ astronomical observations well. The equation of dampened oscillations is [22]:

$m \cdot \ddot{x}+\gamma \cdot \dot{x}+k x=0$ (eq. 14)

where $\mathrm{x}$ is the displacement from equilibrium, $\mathrm{m}$ is the system mass, $\mathrm{k}$ is the elasticity constant and $\mathrm{b}$ is the damping coefficient. Due to red shift of light, the angular velocity, , tends to infinity but very slowly and can be written as follows:

$\omega=\sqrt{\omega_{0}^{2}-\left(\frac{\gamma 1}{2}\right)^{2}}$ (eq. 15)

where $\gamma 1$ is virtually constant because light travels hundreds of thousands of light years before angular velocity changes significantly. Angular velocity $\omega$ of dampened oscillations is constant only when $\gamma 1=\gamma$, i.e., the damping coefficient is a constant. Then the energy of the oscillator is given by:

$E(t)=E(0) e^{-\gamma \cdot t /(2 m)} \quad($ eq. 16)

Compare eq. 7 and 16. Eq. 7 expresses the energy of a dampened oscillator as a function of time. 




\section{CONCLUSIONS}

The version of tired light based on the idea that cosmic red shift is caused by processes strictly confined inside photons agrees with all astronomical observations and had been also validated by research on gravitational properties of laser beams. Being perfectly compatible with an infinite and eternal Universe, this theory easily explains "Superclusters", "Great Walls", "Great Attractors", and the lack of space curvature. Unlike Big Bang, this version of Tired Light does not violate Occam's' razor, does not require ad hoc patches like the assumption that laws of physics change with time, the inflation period and field, dark matter and dark energy.

\section{REFERENCES}

1. Pogge, Richard W. "Essay: The Folly of Giordano Bruno" February 4, 2014 (http://www.astronomy.ohiostate.edu/ pogge/Essays/Bruno.html)

2. Hubble, E. A relation between distance and radial velocity among extra-galactic nebulae, Proceedings of the National Academy of Sciences of the United States of America, vol. 15, issue. 3, March 15 1929, pp. 168-173.

3. Steinhardt and Turok, N, Endless Universe, Beyond the Big Bang, Doubleday, 2007, pp. 7, 13, 150-167.

4. Carr, B, editor, Universe or Multiverse, Cambridge University Press, 2007, Pp. 29-41, 57-240.

5. Tsujikawa, S., Introductory review of cosmic inflation, lecture notes given at The Second Tah Poe School on Cosmology "Modern Cosmology", Naresuan University, Phitsanulok, Thailand, April 17 -25, 2003.

6. Zalta, E.N, Nodelman, U et al, Simplicity, Stanford Encyclopedia of Philosophy, December 202016.

7. Zwicky, F. 1929. On the Red Shift of Spectral Lines through Interstellar Space. Proceedings of the National Academy of Sciences of the United States of America, vol. 15, issue 10, 773-779.

8. Evans, Myron W. and Vigier, J. P. , The Enigmatic Photon: Theory and Practice of the B3 Field. Springer. 1996, p. 29.

9. Lubin, L. M. and Sandage, A, "The Tolman Surface Brightness Test for the Reality of the Expansion. IV. A Measurement of the Tolman Signal and the Luminosity Evolution of Early-Type Galaxies," Astronomical Journal, 2001, vol. 122, pp. 1084-1103.

10. Lubin, L. M. and Sandage, A, "The Tolman Surface Brightness Test for the Reality of the Expansion. II. The Effect of the Point-Spread Function and Galaxy Ellipticity on the Derived Photometric Parameters," Astronomical Journal 2001, vol. 121 pp. 2289-2300.

11. Lubin, L. M. and Sandage, A,, "The Tolman Surface Brightness Test for the Reality of the Expansion. III. Hubble Space Telescope Profile and Surface Brightness Data for Early-Type Galaxies in Three High-Red shift Clusters," Astronomical Journal 2001, vol. 122 pp. 1071-1083.

12. Bonvin, V., Courbin, F. et al, New COSMOGRAIL time delays of HE 0435-1223: H0 to 3.8 per cent precision from lensing in a flat ACDM model, Monthly Notices of the Royal Astronomical Society, November 222016.

13. Woosley, S. E., Kasen, D. et al, Type la Supernova Light Curves, The Astronomical Journal, vol. 662, pp: 487 503, June 102007.

14. Page, N. D., Particle emission rates from a black hole: Massless particles from an uncharged, non-rotating hole, Physical Review, D, vol., 13, p. 198, January 151976.

15. Einstein, A., "Die Grundlage der allgemeinen Relativtätstheorie”, Annalen der Physic, t49, p. 769, 1916.

16. Fock, V.A., "Teoria Spatiului, Timpului si Gravitatiei”, Editura Academiei Romane, Bucuresti, 1962, pp. 47, 176, 178, 193, 201, 247, 299, 300.

17. Rancourt, L. Effect of Light on Gravitational Attraction, Physics Essays, December 2011, vol. 24, No. 4, pp. 557561.

18. Ratzel, D., Wilkens, M. and Menzel, R., Gravitational properties of light - the gravitational field of a laser pulse, New Journal of Physics, Vol. 18, 023009, January 29, 2016.

19. Bonnor, W. B., The gravitational field of photons, General Relativity and Gravitation, vol. 41, January 2009, pp. 77-85.

20. Blanchet L., Damour T., Bala R. I., Clifford M. W., Wiseman A. G., "Gravitational-Radiation Damping of Compact Binary Systems to Second Post-Newtonian Order”, In Physical Review Letters, Volume 74, Number 18, p.3515, 1 May 1996.

21. Abbott, B. P., et. al, Observation of Gravitational Waves from a Binary Black Hole Merger, Physical Review Letters, No. 116, 061102, February 112016.

22. Martin McCall, Classical Mechanics: From Newton to Einstein: A Modern Introduction, $2^{\text {nd }}$ edition, Willey, 2011, pp. 44-50. 


\section{Author' biography with Photo}

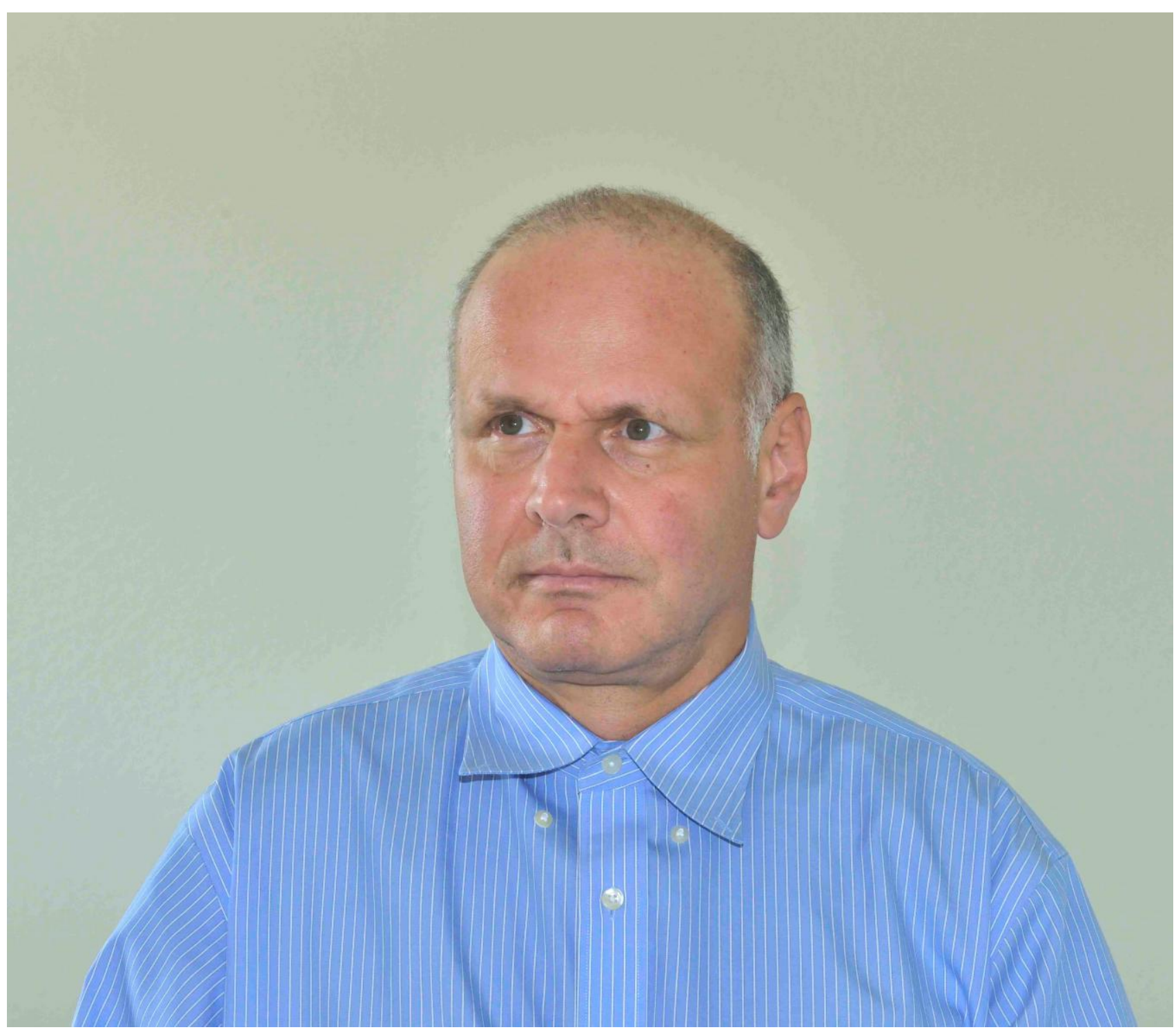

Dr. Dan Brasoveanu is a USA scientist and engineer born in Bucharest Romania in 1954. He has a Ph. D. and a M.S. in Chemical Physics from the University of Maryland, USA and a M.S. in Aeronautical Engineering from the Polytechnic Institute of Bucharest, Romania. He emigrated to the USA in 1986. He worked for Computer Sciences Corporation, Raytheon and Systems Engineering Group of Telephonics on tasks for NASA, Domestic Nuclear Detection Office, US Navy and Department of Defense. He has researched advanced satellite sensors, attitude determination algorithms, radiation sensors, missile and radar systems. He has published more than 50 science papers on combustion phenomena, asvanced aircraft and spaceship design, satellite instruments and attitude determination systems and a book on Quantum Mechanics and Relativity. He has a patent on data compression for HDTV and a patent on a system of cooling with liquid for gas turbines.

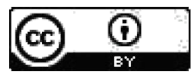

This work is licensed under a Creative Commons Attribution 4.0 International License.

DOI : 10.24297/jap.v13i7.6272 